\title{
Spiral enteroscopy: prospective U.S. multicenter study in patients with small-bowel disorders
}

\author{
Douglas Morgan, MD, MPH, Bennie Upchurch, MD, Peter Draganov, MD, Kenneth F. \\ Binmoeller, MD, Oleh Haluszka, MD, Sreeni Jonnalagadda, MD, Patrick Okolo, MD, lan \\ Grimm, MD, Joel Judah, MD, Jeff Tokar, MD, and Michael Chiorean, MD \\ Chapel Hill, North Carolina; Cleveland, Ohio; Indianapolis, Indiana; Gainesville, Florida; San \\ Francisco, California; Philadelphia, Pennsylvania; Saint Louis, Missouri; Baltimore, Maryland, \\ USA
}

\begin{abstract}
Background-The performance characteristics of spiral enteroscopy have not been welldescribed.
\end{abstract}

Objective-To determine the technical performance, diagnostic and therapeutic yields, and safety of oral spiral enteroscopy in patients with suspected or established small-bowel pathology.

Design-Prospective, multicenter, cohort study, with centralized database.

Setting-Ten U.S. tertiary-care medical centers.

Patients-This study involved 148 participants, of whom 101 were referred for obscure bleeding. All participants referred for antegrade deep enteroscopy were considered eligible.

Intervention-Spiral enteroscopy.

Main Outcome Measurements-Examination duration, depth of insertion, spiral enteroscopy findings, mucosal assessment upon withdrawal, and patient symptom assessment (day 1 and day 7 after the procedure).

Results-Spiral enteroscopy was successful in $93 \%$ of patients, with a median depth of insertion beyond the angle of Treitz of $250 \mathrm{~cm}$ (range 10-600 cm). The mean ( \pm standard deviation) total procedure time was $45.0 \pm 16.2$ minutes for all procedures, and 35.4 minutes for diagnostic procedures. The diagnostic yield was $65 \%$, of which $48 \%$ revealed more than one abnormality. The most common findings were angiectasias $(61.5 \%)$, inflammation $(7.5 \%)$, and neoplasia $(6.8 \%)$. Argon plasma coagulation ablation accounted for $64 \%$ of therapeutic interventions.

Copyright (@) 2010 by the American Society for Gastrointestinal Endoscopy

Reprint requests: Douglas Morgan, MD, MPH, University of North Carolina, Chapel Hill, BioInformatics Bldg, 130 Mason Farm Rd, Suite 4143, Chapel Hill, NC 27599-7080.

Current Affiliations: (D.M., I.G.), University of North Carolina, Chapel Hill, North Carolina, (M.C.), Indiana University School of Medicine, Indianapolis, Indiana, (B.U.), Creighton University, Omaha, Nebraska, (P.D., J.J.), University of Florida, Gainesville, Florida, (K.F.B.), California Pacific Medical Center, San Francisco, California, (O.H., J.T.), Fox Chase Cancer Center, Philadelphia, Pennsylvania, (S.J.), Washington University at Saint Louis, Saint Louis, Missouri, (P.O.), Johns Hopkins University, Baltimore, Maryland.

Presented at Digestive Disease Week, May 30-June 4, 2009, Chicago, Illinois. (Gastrointest Endosc 2009;69:AB127-8).

DISCLOSURE: Study logistical support was received from Spirus Medical, Inc (Stoughton, Mass). D. Morgan, B. Upchurch, P. Dragnov, K. Binmoeller, O. Haluszka, S. Jonnalagadda, P. Okolo, I. Grimm, and M. Chiorean received honoraria for teaching and received support for the current clinical trial from Spirus Medical, Inc. Study biostatistical support (D.M.) was facilitated through the University of North Carolina Center for Gastrointestinal Biology and Disease (P30 DK 034987). No other disclosures of financial relationships relevant to this publication were made. 
Limitations-This was not a randomized, controlled trial of deep enteroscopy modalities.

Conclusion-Spiral enteroscopy appears to be safe and effective for evaluation of the small bowel. The procedure duration, depth of insertion, and diagnostic and therapeutic yields compare favorably with previously published data on other deep enteroscopy techniques such as singleballoon and double-balloon enteroscopy. Comparative studies are warranted. (Gastrointest Endosc 2010;72:992-8.)

The advent of deep enteroscopy has revolutionized the management of patients with midsmall-bowel diseases. Since the introduction of double-balloon enteroscopy by Yamamoto in 2001, two additional techniques have become available, single-balloon enteroscopy and spiral enteroscopy. ${ }^{1-3}$ Double-balloon enteroscopy and single-balloon enteroscopy entail a similar mechanism of advancement consisting of sequential bowel pleating by a push-pull technique that uses a balloon-fitted overtube with or without a second balloon inserted over the tip of a dedicated enteroscope.

In contrast, spiral enteroscopy, or rotational enteroscopy, uses a spiral or raised helix-fitted overtube coupled with the enteroscope, advanced as a unit into the small bowel by continuous rotation of the overtube in a manner similar to use of a corkscrew. An inner sleeve allows the independent motion of the overtube from the enteroscope during advancement and withdrawal. The main difference between balloon enteroscopy and spiral enteroscopy is that the latter uses a more or less continuous pleating of the small bowel by a clockwise rotation of the overtube rather than the push-pull technique of the former two methods. ${ }^{4}$ Previous small studies have suggested that spiral enteroscopy allows advancement into the small bowel more efficiently than do techniques using balloon-assisted devices. ${ }^{5}$ The technical performance of spiral enteroscopy as well as the diagnostic and therapeutic yields have not been rigorously studied.

The aims of the present study were to determine the performance, yield, and safety of oral spiral enteroscopy in patients with suspected or established small-bowel pathology within the framework of a prospective, multicenter study in the United States.

\section{MATERIALS AND METHODS}

\section{Study design}

The study design consisted of a prospective, multicenter investigation of antegrade spiral enteroscopy yield and efficacy. A centralized database was used to collect data from patients evaluated at participating U.S. centers with experience in balloon enteroscopy. Patients with suspected or established small-bowel disorders referred for anterograde deep enteroscopy were considered eligible for the study if they had a previous nondiagnostic upper and lower endoscopy or if they had other objective evidence of small-bowel disease based on prior capsule endoscopy or small-bowel radiological studies. Patients with histories of esophageal stricture, varices, advanced cirrhosis, or coagulopathy were excluded. Per study protocol, centers with an enrollment of fewer than 10 participants were not included in the analysis. Research informed consent was obtained from all patients before the procedure.

\section{Procedure and instruments}

The spiral enteroscopy technique has been described. ${ }^{4}$ Each procedure was performed by a two-physician team, consisting of two experienced gastroenterologists or one faculty gastroenterologist and one gastroenterology fellow. One physician guided the enteroscope while the other operated the overtube. All investigators had participated in or observed at least 20 spiral enteroscopy procedures before the current investigation. 
The Discovery small-bowel overtube (DSB; Spirus Medical, Inc, Stoughton, Mass) was used for all study procedures. The use of the DSB standard profile or DSB low-profile system was determined based upon patient characteristics and physician preference. The choice of enteroscope was made based upon site availability, including the Fujinon diagnostic double-balloon enteroscope (EN-450TS; Fujinon, Inc, Saitama, Japan) or the Olympus single-balloon enteroscope (SIF-Q180; Olympus Corporation, Tokyo, Japan).

All sites chose procedure sedation according to local standards and anesthesia guidelines for individual patients. Mallampati scores were assessed by the anesthesia or gastroenterology attending physician. The use of fluoroscopy was allowed at the discretion of the site gastroenterologist.

\section{Study endpoints}

Procedure times-Study times were recorded by the study staff during each procedure, with the total procedure time (enteroscope insertion to enteroscope removal) as the primary time endpoint. The following time milestones were also measured: helix engagement of the pylorus and duodenum, maximum overtube insertion, maximum enteroscope insertion, and procedure termination. The times of each therapeutic intervention were recorded and summed to facilitate calculation of the total therapy time. Interventions included vascular lesion ablation, polypectomy, stricture dilation, and solution injection (eg, India ink). The procedure-adjusted diagnostic time was defined as the total procedure time minus the total therapy time, which was calculated on a per-participant basis.

Depth of insertion and procedure yields-The depth of maximum enteroscope insertion was estimated for each case, based upon withdrawal or bowel unpleating in sequential $10-\mathrm{cm}$ increments, a variation on the technique described by May et al, ${ }^{6}$ and estimated as total centimeters beyond the angle of Treitz. The overtube external helix facilitates controlled advancement and withdrawal of the enteroscope, such that the bowel slippage observed with balloon enteroscopy was not a factor in depth estimations. Procedure success was defined as enteroscope advancement beyond the angle of Treitz.

The procedure diagnostic and therapeutic yields were calculated based upon procedure findings and therapeutic interventions. Correlation with capsule endoscopy findings was graded as definite, probable, or negative.

Safety and tolerance-The evaluation of mucosal changes was systematically performed upon enteroscope and overtube withdrawal. Assessment of the mucosa upon withdrawal of the enteroscope is standard, but for the most part, other studies have not commented on the spectrum of iatrogenic mucosal or bowel-wall damage. Specific evaluation focused upon the following anatomic regions: proximal and distal esophagus, gastric lesser curve, pylorus, second duodenum, angle of Treitz, and small bowel. Enteroscopy-related trauma was graded as none/mild, moderate, and mucosal disruption. The type and severity of side effects and complications were assessed during and after the procedure as well as at postprocedure days 1 and 7 by telephone interview. Patients were specifically queried about throat discomfort, difficult or painful swallowing, abdominal discomfort or distention, and ability to resume normal activities after the procedure. Preprocedure assessment of chronic abdominal symptoms by Rome III or other criteria was not performed. ${ }^{7}$

A centralized, standard database, with Web-based data entry was used. The database included 185 fields within the categories of demographics, medical history, enteroscopy indications, procedure efficacy, therapeutic interventions, mucosal trauma, patient followup, physician experience, and impressions. Statistical analysis was performed with STATA 
9 (Stata Corporation, College Station, Tex). The study was approved by the institutional review boards of each center.

\section{RESULTS}

A total of 149 participants with suspected or established small-bowel disorders were enrolled at 10 U.S. centers from April 2008 through October 2008. The median age was 68.0 years (range $20-88$ years), and $40 \%$ were male $(n=57)$. The current analysis included 141 patients who had complete data. In agreement with the study protocol, centers with an enrollment of fewer than 10 participants at the time of study closure were not included in the analysis; 3 participants (two centers) were thereby excluded, each of whom had an uncomplicated study and usual procedure time (37, 45, and 51 minutes). Five procedures were aborted before initiation or completion - two for medical indications and two in patients with obstructive sleep apnea (the anesthesiologist recommended intubation, but the participants declined). One case was instructive, wherein a previously undiagnosed, subtle cervical web was encountered; the spiraling of the overtube helix did not facilitate advancement but rather bowing of the overtube.

The enrolled patient population had a significant percentage of comorbidities, which reflects the obscure GI bleeding population in the United States (Table 1). This included coronary artery disease (36\%), congestive heart failure (11\%), chronic obstructive pulmonary disease $(14 \%)$, diabetes $(32 \%)$, and hypertension (52\%). Prior abdominal or pelvic surgery was common, noted in $52 \%$ of participants. A documented history of intra-abdominal adhesions was reported in $8.5 \%(n=12)$ of patients. Although the condition was not exclusionary, no patients with prior Nissen fundoplication were enrolled. The use of chronic anticoagulant medication before the procedure was common, including aspirin (27\%), clopidogrel (12\%), warfarin (6.4\%), and nonsteroidal anti-inflammatory drugs (5.6\%). The mean ( \pm standard deviation [SD]) patient body mass index was $28.8 \pm 9.0 \mathrm{~kg} / \mathrm{m}^{2}$, with a range of 15.6 to 69.4 $\mathrm{kg} / \mathrm{m}^{2}$.

\section{Procedure indications}

Obscure GI bleeding was the indication for deep enteroscopy in nearly three-fourths of patients $(\mathrm{n}=101,72 \%)$. Of those cases with obscure GI bleeding, $32 \%$ were overt and $68 \%$ occult (stool occult blood test positive or iron deficiency anemia). Two-thirds of this obscure GI bleeding cohort $(n=67)$ had received transfusions within 6 months of the procedure.

Prior diagnostic evaluations included EGD (83\%), colonoscopy (89\%), push enteroscopy (26\%), and balloon enteroscopy ( $8.5 \%)$ (Table 2). The majority of participants $(\mathrm{n}=113)$ had undergone capsule endoscopy before enrollment, and 79\% had abnormal results. Abnormal imaging results also were a common indication for spiral enteroscopy, including abdominal CT $(n=22)$, small-bowel follow-through $(n=5)$, and other imaging $(n=8)$. There were no documented prior diagnostic or therapeutic angiography evaluations.

\section{Procedure efficacy}

Spiral enteroscopy was successful in 93\% (132 of 142) of cases, with advancement beyond the proximal jejunum and the presumptive extent of push enteroscopy. In 6 cases, only the proximal jejunum was accessed, and in 4 cases, the angle of Treitz could not be reached, including the patient with the cervical web. The use of fluoroscopy was limited to 16 cases $(11 \%)$. The standard profile DSB overtube was used in nearly all cases (93\%). The Olympus and Fujinon enteroscopes were used in $68 \%$ and $32 \%$ of cases, respectively, according to site availability and preference. Most procedures were performed with patients under 
moderate conscious sedation with a combination of midazolam, fentanyl, or meperidine and propofol administered either by an anesthesiologist or qualified endoscopy nursing staff.

The median depth of insertion beyond the angle of Treitz, estimated as described in Materials and Methods, was $250 \mathrm{~cm}$ (mean $\pm \mathrm{SD}=250.3 \pm 94.6 \mathrm{~cm}$; range 10-600 cm). The frequency plot of depth estimations demonstrates a gaussian distribution, an expected result given variable patient anatomy and also arguing against significant bias in the depth estimations (Fig. 1). The terminal ileum was reached in one case, in a patient with a Rouxen-Y anastomosis with an estimated $100 \mathrm{~cm}$ of small bowel excluded.

The median total procedure time was 43.0 minutes (range 21-104 minutes). The mean therapeutic intervention time was 11.4 minutes (range 0-73 minutes). The mean $( \pm \mathrm{SD}$ ) adjusted diagnostic procedure time for all procedures was $34.4 \pm 10.1$ minutes, calculated for each patient. Importantly, this estimation was in agreement with the total procedure time in nontherapeutic cases (35.4 minutes, $\mathrm{n}=12$ ). The mean $( \pm \mathrm{SD})$ times to helix engagement beyond the pylorus, maximum DSB overtube insertion time, and maximum enteroscope insertion time were $8.4 \pm 5.9$ minutes, $22.1 \pm 11.5$ minutes, and $25.7 \pm 12.1$ minutes, respectively.

\section{Diagnostic and therapeutic yields}

Abnormalities were detected at spiral enteroscopy in $65.2 \%(n=92)$ of the evaluations, of which $44 \%$ were felt to be definite findings and $21 \%$ probable findings (Table 3 ). The most common diagnostic findings in those with positive study results included vascular ectasias (56.2\%), inflammation (15\%), and neoplasia (7.9\%). For study purposes, inflammation included erosions, ulcerations, and/or mucosal changes suggestive of Crohn's disease, such as thickened or denuded villi, mucosal erythema and edema, or fold thickening with or without associated strictures. The majority of these diagnostic findings were found in the mid-jejunum (48 of 82). The correlation with capsule endoscopy findings, according to protocol grading, was $40 \%$ definite, $32 \%$ probable or possible, and $28 \%$ negative. Ablation with argon plasma coagulation of vascular ectasias accounted for the majority $(64 \%)$ of therapeutic interventions. Resection of polyps and mucosal biopsy were performed in $16 \%$ of patients.

\section{Procedure-related mucosal changes}

Systematic assessment of mucosal changes related to the enteroscope and DSB overtube in each segment of the small bowel and intestines was performed upon withdrawal. Mucosal changes were limited in the majority of procedures (Table 4). Mucosal disruption was noted in 8 cases, including small intestine (2), duodenum (1), pylorus (3), and proximal esophagus (2). There were no perforations. The areas in which the mucosal changes occurred were consistent among centers and operators, as assessed by significant erythema and mucosal disruption (angle of Treitz, pylorus, esophagus).

\section{Adverse events and side effects}

Physicians judged the overall procedure as well-tolerated (77\%), fairly well-tolerated $(14.5 \%)$, or average $(5 \%)$. A small number (3.5\%) of cases were poorly tolerated.

Significant symptoms during the procedure were limited but included agitation (1.4\%), significant cough (1.4\%), and transient stridor (2\%). One transient intussusception of the pleated bowel was observed, with rapid resolution by gentle manipulation of the bowel by using the enteroscope.

The most common procedure side effects at 24 hours included sore throat (28.3\%) and/or swallowing discomfort (24\%) and abdominal bloating or discomfort (19\%). Residual side 
effects at 7 days included sore throat (0.7\%), swallowing discomfort (2.2\%), and abdominal bloating or discomfort (6.7\%). There were no serious adverse events recorded such as perforation, pancreatitis, ileus, or death. Ten office visits were recorded during the week after the spiral enteroscopy.

\section{DISCUSSION}

Our study shows that spiral enteroscopy is a safe and effective deep enteroscopy technique. The mean procedure time (mean endoscope insertion to removal time) of 45 minutes compares favorably with the other deep enteroscopy approaches. ${ }^{8-13}$ In the U.S. multicenter, double-balloon enteroscopy study, the mean procedure duration was $92.1 \pm 37$ minutes ( $\mathrm{n}=$ 114). ${ }^{9}$ In particular, the time to maximal advancement of the enteroscope (mean $25.7 \pm 12$ minutes) is efficient, suggesting that the continuous pleating of the small bowel by using a clockwise rotation of the helix overtube may be a more efficient advancement method than the push-pull technique used with balloon-assisted enteroscopy. As noted, the terminal ileum was reached in one patient with a gastric bypass, suggesting efficient pleating of the small bowel. We acknowledge that our depth of insertion estimation method has not been adequately validated, and therefore a comparison with historic cohorts may not represent an adequate control group.

The diagnostic and therapeutic yields of the procedures in this study compare favorably with data previously published regarding balloon-assisted enteroscopy. As anticipated in these patient populations, the most common indication for spiral enteroscopy was obscure GI bleeding, and the most common finding was vascular malformations. It is worth mentioning that all endoscopists participating in this study had previously performed balloon-assisted enteroscopies, yet the overall impression was that spiral enteroscopy was a very stable platform for interventions such as cautery, biopsy, polypectomy, or solution injection. One potential technical limitation with the single-balloon enteroscopy device is that air can escape from the bowel distal to the enteroscope through the space between the endoscope shaft and the overtube. This was not thought to be the case with spiral enteroscopy because of the locking device in the overtube handle. Clearly, more comparative studies are required to further elucidate the performance aspect of this procedure. The aims of the present study were to determine the performance and yield of spiral enteroscopy in the small bowel. Therefore, the prevalence of significant findings within the reach of standard endoscopy (such as stomach, duodenum, or colon) was not consistently reported.

Despite the high prevalence of serious comorbid conditions including chronic obstructive pulmonary disease, coronary artery disease, congestive heart failure, and diabetes, the procedure was well-tolerated by the majority of patients, with a relatively limited incidence of side effects such as sore throat, dysphagia, or odynophagia. There were no serious complications such as pancreatitis, perforation, bleeding, or infection. The incidence of significant mucosal trauma was measureable ( 8 mucosal disruptions were noted), in contrast to the experience in the U.S. double-balloon enteroscopy study of similar design, which included one mucosal tear. ${ }^{9}$ It was observed that the mucosal changes in the esophagus were often related to the depth of sedation at procedure initiation. Only one patient could not be intubated with the overtube, this because of an unrecognized cervical web. This compares favorably with data published with alternative deep enteroscopy techniques. ${ }^{9,12,14-17}$

It is noteworthy that most procedures in the study were performed under deep sedation with intravenous propofol. A small proportion of procedures were performed with the patient under moderate sedation or general anesthesia. In the latter case, we noted that allowing the endotracheal tube to deflate at the beginning of the procedure facilitates intubation of the esophagus, with minimal resistance and minimal or no mucosal trauma. 
The prospective study design, with the use of a centralized database, strengthened the validity of our study data. The patient population is representative of individuals with obscure GI bleeding and, in general, patients in the United States requiring deep enteroscopy. In addition, the evaluation of mucosal changes upon enteroscope withdrawal was systematically performed to capture the spectrum of iatrogenic mucosal changes.

We acknowledge that this study was not a randomized trial with a comparative arm with capsule endoscopy, push enteroscopy, or other deep-enteroscopy modalities. Assessment of the learning curve was not included because all gastroenterologists had prior experience with balloon enteroscopy and spiral enteroscopy. Although the study population was representative of the U.S. obscure GI bleeding population, patients with upper GI strictures and severe liver disease were excluded. We also were not able to evaluate long-term clinical outcomes such as improvement or cessation of bleeding and survival. The study may have had limited power to detect infrequent serious complications (eg, pancreatitis, perforation). Last, this study did not evaluate retrograde spiral enteroscopy or spiral enteroscopy-assisted ERCP.

What are the potential limitations of spiral enteroscopy (Table 5)? The procedure efficiency is balanced by the overtube diameter $(16 \mathrm{~mm})$ in this "first generation" version of the technology. This may increase the number of procedure-related mucosal changes and the anesthesia requirements. We observed one patient who could not be intubated with the spiral overtube because of a cervical web. The areas of procedural mucosal changes and mucosal disruption were similar among centers, which suggests that these are expected "pressure points" in spiral enteroscopy and that technique or overtube modifications and physician experience may serve for prevention. In addition, the current spiral overtube technique requires two operators - two gastroenterologists or a gastroenterologist and an assistant. Importantly, latex allergy is not an issue with the spiral overtube.

In this initial study of spiral enteroscopy by endoscopists experienced in deep enteroscopy at tertiary-care U.S. centers, spiral enteroscopy by the oral approach appears safe in selected patients and can be used for diagnostic and therapeutic maneuvers in the mid-bowel and possibly the proximal ileum. The performance and procedure duration appear comparable with those of alternative deep-enteroscopy techniques. Further comparative prospective studies are warranted to analyze outcomes such as procedure yields, transfusion burden, and survival.

\section{Acknowledgments}

The authors acknowledge study biostatistical support from Paris Heidt, Chris Martin, Ginny Sharpless, and Mark Duncan of the University of North Carolina Center for Gastrointestinal Biology and Disease. The authors also acknowledge Paul Ackerman, David Johnson, Doug Pleskow, and Debra Helper for scientific input and Tony Resendes, Bill Bookwalter, Frank Guilfoyle, and Sean McKelvey of Spirus Medical, Inc for study logistical support.

\section{Abbreviation}

DSB Discovery small-bowel overtube

\section{References}

1. Yamamoto H, Sekine Y, Sato Y, et al. Total enteroscopy with a nonsurgical steerable doubleballoon method. Gastrointest Endosc. 2001; 53:216-20. [PubMed: 11174299]

2. Sunada K, Yamamoto H. Technology and indications. Gastrointest Endosc Clin N Am. 2009; 19:325-33. [PubMed: 19647642] 
3. Gerson LB. Capsule endoscopy and deep enteroscopy: indications for the practicing clinician. Gastroenterology. 2009; 137:1197-201. [PubMed: 19703454]

4. Akerman PA, Cantero D. Spiral enteroscopy and push enteroscopy. Gastrointest Endosc Clin N Am. 2009; 19:357-69. [PubMed: 19647645]

5. Akerman PA, Agrawal D, Cantero D, et al. Spiral enteroscopy with the new DSB overtube: a novel technique for deep peroral small-bowel intubation. Endoscopy. 2008; 40:974-8. [PubMed: 19065477]

6. May A, Nachbar L, Schneider M, et al. Push-and-pull enteroscopy using the double-balloon technique: method of assessing depth of insertion and training of the enteroscopy technique using the Erlangen Endo-Trainer. Endoscopy. 2005; 37:66-70. [PubMed: 15657861]

7. Longstreth GF, Thompson WG, Chey WD, et al. Functional bowel disorders. Gastroenterol. 2006; 130:1480-91.

8. Di Caro S, May A, Heine DG, et al. The European experience with double-balloon enteroscopy: indications, methodology, safety, and clinical impact. Gastrointest Endosc. 2005; 62:545-50. [PubMed: 16185969]

9. Mehdizadeh S, Ross A, Gerson L, et al. What is the learning curve associated with double-balloon enteroscopy? Technical details and early experience in 6 U.S. tertiary care centers. Gastrointest Endosc. 2006; 64:740-50. [PubMed: 17055868]

10. Gerson LB, Batenic MA, Ross A, et al. Long-term outcomes after double balloon enteroscopy for obscure gastrointestinal bleeding. Clin Gastroenterol Hepatol. 2009; 7:664-9. [PubMed: 19514115]

11. Ramchandani M, Reddy DN, Gupta R, et al. Diagnostic yield and therapeutic impact of singleballoon enteroscopy: series of 106 cases. J Gastroenterol Hepatol. 2009; 24:1631-8. [PubMed: 19686408]

12. Gross SA, Stark ME. Initial experience with double-balloon enteroscopy at a U.S. center. Gastrointest Endosc. 2008; 67:890-7. [PubMed: 18178204]

13. Kawamura T, Yasuda K, Tanaka K, et al. Clinical evaluation of a newly developed single-balloon enteroscope. Gastrointest Endosc. 2008; 68:1112-6. [PubMed: 18599052]

14. Gerson LB. Outcomes associated with deep enteroscopy. Gastrointest Endosc Clin N Am. 2009; 19:481-96. [PubMed: 19647653]

15. Hegde SR, Iffrig K, Tianyu L, et al. Double balloon enteroscopy in the elderly: safety, findings, and diagnostic and therapeutic success. Gastrointest Endosc. 2010; 71:983-9. [PubMed: 20189563]

16. Upchurch BR, Sanaka MR, Lopez AR, et al. The clinical utility of single-balloon enteroscopy: a single center experience of 172 procedures. Gastrointest Endosc. 2010; 71:1218-23. [PubMed: 20409544]

17. Frantz DJ, Dellon ES, Grimm IS, et al. Single-balloon enteroscopy: results from an initial experience at a U.S. tertiary-care center. Gastrointest Endosc. 2010; 72:422-6. [PubMed: 20541189] 


\section{Take-home Message}

- Spiral enteroscopy appears to be safe and effective for evaluation of the small bowel. The procedure duration, depth of insertion, and diagnostic and therapeutic yields compare favorably with other deep enteroscopy techniques such as single-balloon and double-balloon enteroscopy. Comparative studies are warranted. 


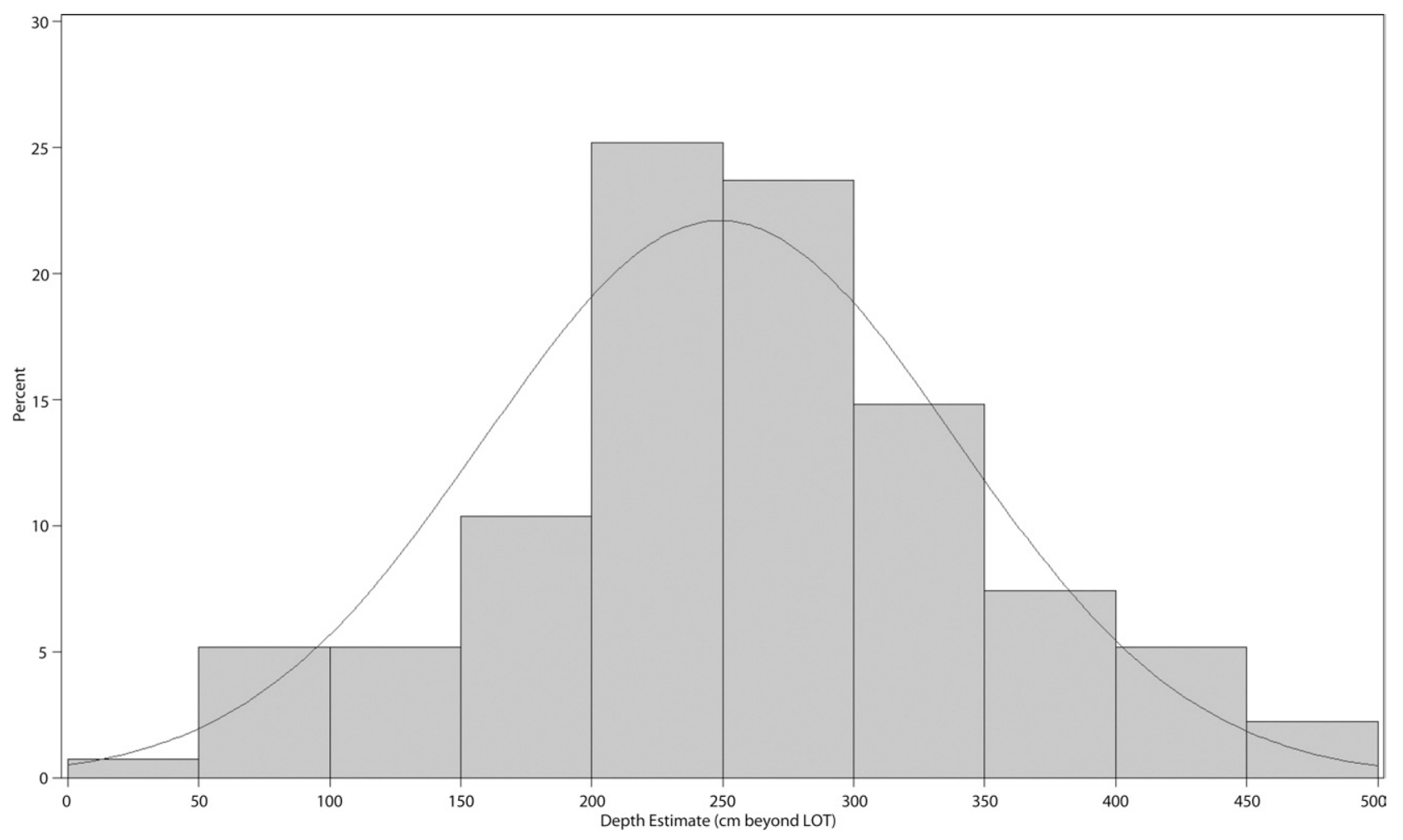

Figure 1.

Spiral enteroscopy: small-bowel maximum depth of insertion beyond the ligament of Treitz (LOT). Spiral enteroscopy was successful in $93 \%$ of cases, with advancement beyond the proximal jejunum and the presumptive extent of push enteroscopy. In 4 patients, the angle of Treitz could not be reached, including a patient with a cervical web. In 6 patients, the examination was limited to the proximal jejunum. The maximum depth of insertion estimates, expressed in centimeters, demonstrate a gaussian distribution, fulfilling criteria for normality. 
TABLE 1

Spiral enteroscopy: patient medical and surgical history

\begin{tabular}{|lc|}
\hline Chronic medical condition & No. of patients (\%) \\
\hline Coronary artery disease & $51(36.2)$ \\
\hline Congestive heart failure & $16(11.4)$ \\
\hline Chronic obstructive pulmonary disease & $20(14.2)$ \\
\hline Hypertension & $73(51.2)$ \\
\hline Diabetes & $45(31.2)$ \\
\hline Crohn's disease & $6(4.3)$ \\
\hline Surgical history & \\
\hline Cholecystectomy & $25(17.7)$ \\
\hline Appendectomy & $24(17.0)$ \\
\hline Hysterectomy & $25(17.7)$ \\
\hline Altered gastric anatomy & $3(2.1)$ \\
\hline Intestinal resection, colon or small bowel & $9(6.4)$ \\
\hline
\end{tabular}


TABLE 2

Spiral enteroscopy: antecedent diagnostic evaluations

\begin{tabular}{|lc|}
\hline Diagnostic evaluation & No. $(\%)$ \\
\hline Endoscopy & \\
\hline Capsule endoscopy & $113(79 \%)^{*}$ \\
\hline Upper endoscopy & $117(83.0)$ \\
\hline Colonoscopy & $124(88.0)$ \\
\hline Push enteroscopy & $37(26.2)$ \\
\hline Balloon enteroscopy & $12(8.5)$ \\
\hline Radiology & \\
\hline Abdominal CT, abnormal result & $22(15.6)$ \\
\hline Small-bowel follow-through & $17(12.1)$ \\
\hline Enterography (CT, MR) & $8(5.7)$ \\
\hline
\end{tabular}

* Eighty-nine of the 113 antecedent capsule endoscopy results were characterized as abnormal. 
TABLE 3

Spiral enteroscopy: summary of small-bowel findings

\begin{tabular}{|lccc|}
\hline & Angiectasia & Neoplasia & Inflammation \\
\hline Upper GI tract & 21 & 3 & 6 \\
\hline Duodenum, distal & 9 & 2 & 0 \\
\hline Jejunum & & & \\
\hline Proximal & 24 & 1 & 4 \\
\hline Middle & 33 & 0 & 6 \\
\hline Distal & 18 & 4 & 2 \\
\hline Ileum & 4 & 2 & 2 \\
\hline Total & 109 & 10 & 20 \\
\hline
\end{tabular}

Upper GI tract includes findings in esophagus, stomach, and/or proximal duodenum. Inflammation refers to mucosal erythema, congestion, thickened folds, thick or denuded villi, ulceration, erosions, and/or erythema. The characterizations of small- bowel segments are gross estimations for lesion localization. 
TABLE 4

Spiral enteroscopy: mucosal changes

\begin{tabular}{|lccc|}
\hline & Erythema, none or minimal (\%) & Erythema, moderate (\%) & Mucosal disruption (no.) \\
\hline Esophagus, proximal & 85 & 15 & 2 \\
\hline Esophagus, distal & 66 & 34 & 0 \\
\hline Gastric, lesser curve & 93 & 6.7 & 1 \\
\hline Gastric, pylorus & 75 & 25 & 3 \\
\hline Duodenum & 80 & 20 & 0 \\
\hline Angle of Treitz & 84 & 16 & 2 \\
\hline Small bowel & 79 & 21 & 0 \\
\hline
\end{tabular}


TABLE 5

Comparison of deep enteroscopy techniques, U.S. experience

\begin{tabular}{|cccc|}
\hline & Double balloon $^{\mathbf{9 *}^{*}, \mathbf{1 2 , 1 5}}$ & Single balloon $^{\mathbf{1 6 , 1 7}}$ & Spiral enteroscopy \\
\hline Overtube material & Latex & Silicone & PVC \\
\hline OD/ID, mm & $13.2 / 10.8$ & $13.2 / 11$ & $16 / 9.8$ \\
\hline Length, cm & 135 & 132 & 118 \\
\hline Sedation routine & Deep, propofol & Moderate, conscious & Deep, propofol \\
\hline Insertion depth, cm & $218-370$ & 132 & 250 \\
\hline Duration, minutes & $68-101$ & $38-49$ & 45 \\
\hline
\end{tabular}

$P V C$, Polyvinyl chloride; $O D$, outer diameter; $I D$, inner diameter.

* The U.S. multicenter double-balloon enteroscopy study reported for antegrade double-balloon enteroscopy procedures a mean ( \pm SD) insertion depth of $370 \pm 167 \mathrm{~cm}$, with a mean $( \pm \mathrm{SD})$ procedure duration of $90.3 \pm 37$ minutes. 9 